\title{
Preservation of skin DNA for oligonucleotide array CGH studies: a feasibility study
}

\author{
Mehdi Nassiri • Dijana Gugic · Joseph Olczyk • \\ Sharon Ramos $\cdot$ Vladimir Vincek
}

Received: 30 May 2007 / Revised: 27 June 2007 / Accepted: 10 July 2007 / Published online: 31 July 2007

(C) Springer-Verlag 2007

\begin{abstract}
Array-based comparative genomic hybridization (a-CGH) is a promising tool for clinical genomic studies. However, pre-analytical sample preparation methods have not been fully evaluated for this purpose. Parallel sections of normal male human skin biopsy samples were collected and immediately immersed in saline, formalin and a molecular fixative for 8,12 and $24 \mathrm{~h}$. Genomic DNA was isolated from the samples and subjected to amplification and labeling. Labeled samples were then co-hybridized with normal reference female DNA to Agilent oligonucleotide-based a-CGH 44k slides. Pre-analytic parameters such as DNA yield, quality of genomic DNA and labeling efficacy were evaluated. Also microarray analytical variables, including the feature signal intensity, data distribution dynamic range, signal to noise ratio and background intensity levels were assessed for data quality. DNA yield and quality of genomic DNA - as evaluated by spectrophotometry and gel electrophoresis-were similar for fresh and molecular fixative-exposed samples. In addition, labeling efficacy of dye incorporation was not drastically different. There was no difference between fresh and molecular fixative material comparing scan parameters and stem plot analysis of a-CGH result. Formalin-fixed samples, on the other hand, showed various errors such as oversaturation, non-uniformity in replicates, and decreased signal to noise
\end{abstract}

M. Nassiri · D. Gugic $\cdot$ J. Olczyk · S. Ramos · V. Vincek Department of Pathology, University of Miami, Miller School of Medicine, Miami, FL, USA

M. Nassiri ( $\square)$

Research Molecular Pathology Laboratories, University of Miami, Miller School of Medicine, 1550 NW 10th Ave Papanicolaou Bldg \#411,

Miami, FL 33136, USA

e-mail: mnassiri@med.miami.edu ratio. Overall, the a-CGH result of formalin samples was not interpretable. DNA extracted from formalin-fixed tissue samples is not suitable for oligonucleotide-based a-CGH studies. On the other hand, the molecular fixative preserves tissue DNA similar to its fresh state with no discernable analytical differences.

Keywords Genomics - Array comparative genomic hybridization · Fixatives · Tissue preservation

\section{Introduction}

Applications of new technologies have resulted in major advancements in laboratory medicine [1]. Bringing these advances into clinical practice, however, requires careful evaluation and validation. Array-based comparative genomic hybridization (a-CGH) is an extremely powerful tool that can generate high resolution mapping of chromosomal abnormalities. Advances in microarray technology and bioinformatics have now made a-CGH easily available and affordable [14, 15]. With a-CGH's potential for clinical application, it is important that guidelines for proper sample preparation and control of quality are developed. Since conventional methods of clinical tissue preparation commonly employ formalin fixation, we studied the suitability of formalin for array CGH studies and compared the results to that of clinical samples that were preserved in a newly developed, molecular-friendly fixative.

The study was approved by the University of Miami Institutional Review Board. Three separate normal skin biopsies from one volunteer healthy male were immediately sliced in three parts each $0.1 \times 0.2 \times 0.2 \mathrm{~cm}$. One part was immersed in normal saline solution, one part in a methanol-based molecular tissue fixative, UMFIX (Universal 
Molecular Fixative, marketed as Tissue-Tek ${ }^{\circledR}$ Xpress $^{\mathrm{TM}}$ Molecular Fixative, Sakura Finetek, Torrance, CA), and the third slice was fixed in $10 \%$ neutral buffered formalin. Volume of fixative/preservative was $150 \mathrm{ml}$ and incubation was performed at room temperature. After 8 (set 1), 12 (set 2) and $24 \mathrm{~h}$ (set 3), the genomic DNA was extracted from the samples using Puregene DNA Purification System tissue kit (Gentra, Minneapolis, MN). One microliter of extracted DNA solution was diluted in Tris-EDTA (TE) buffer and evaluated on ND-1000 spectrophotometer (NanoDrop Technologies, Rockland, DE). Gel electrophoresis ( $0.8 \%$ agarose gel) was performed to evaluate the quality of genomic DNA.

DNA yield and quality of genomic DNA as evaluated by spectrophotometry, were similar between the samples (Table 1), although UMFIX samples appeared to have better quality. A260/A280 ratio determines presence of contaminating proteins and a ratio of more than 1.8 is generally considered to be indicative of high quality sample. All UMFIX samples consistently had a ratio of more than 1.8. We further evaluated the quality of DNA by agarose gel electrophoresis that showed presence of high molecular weight (HMW) genomic DNA band in all samples (Fig. 1a). While formalin-fixed sample showed higher degradation and lower intensity of genomic DNA, UMFIXexposed samples did not appear degraded.

Genomic DNA from the skin samples and the control female DNA (Promega, Madison, WI) were then subjected to amplification according to Agilent's (Palo Alto, CA) protocol for oligonucleotide array-based $\mathrm{CGH}$ for genomic DNA (version 2.0 August 2005). Amplification, of both male genomic DNA and female control DNA, $100 \mathrm{ng}$ each, was performed using Qiagen REPLI-g Amplification Kit. Amplified DNA was digested during a $2 \mathrm{~h}$ incubation at $37^{\circ} \mathrm{C}$, with Alu I and Rsa I (10 U/ $\mu \mathrm{l} ; 5 \mu \mathrm{l} /$ reaction) restriction enzymes (Promega, Madison, WI). Purification of digested DNA was performed with QIAprep Spin Miniprep
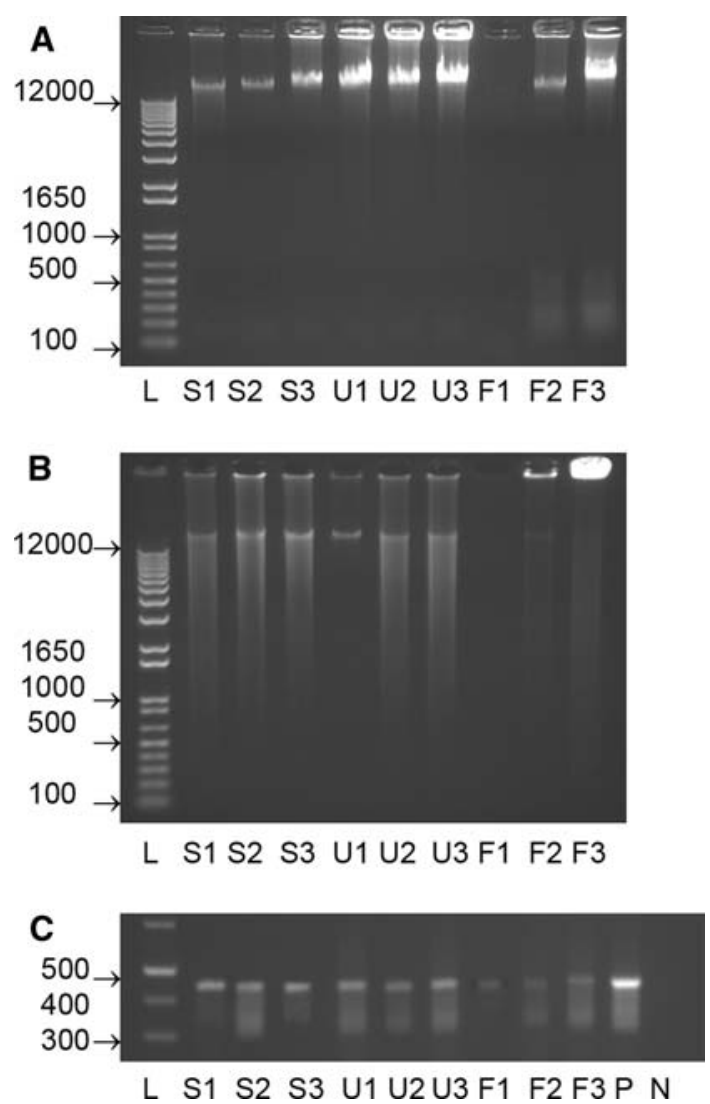

Fig. 1 Result of gel electrophoresis for genomic DNA a HMW genomic DNA is visible in all samples. There is more DNA degradation in formalin-fixed samples. b Formalin-fixed samples do not show uniform strong smear after linear amplification. $\mathbf{c}$ PCR for GAPDH shows a $450 \mathrm{bp}$ amplicon in all samples with a lesser intensity in formalinfixed samples, $L$ ladder (numbers indicate bp), $S$ fresh control in saline, $U$ UMFIX, $F$ formalin, $P$ positive control, $N$ negative control

Kit (Qiagen, Valencia, CA). Digested DNA was subjected to electrophoresis to evaluate quality of amplified DNA by visual inspection of its uniformity and range.
Table 1 Spectrophotometric result of total DNA yield, DNA purity, and labeling efficiency, for fresh, formalin-fixed and UMFIX-exposed samples

\begin{tabular}{llllll}
\hline Sample & $\begin{array}{l}\text { Total DNA } \\
(\mu \mathrm{g})\end{array}$ & A260/A280 & A260/A230 & $\begin{array}{l}\text { Sample-cy5 labeling } \\
\text { efficiency }(\mathrm{pmol} / \mu \mathrm{g})\end{array}$ & $\begin{array}{l}\text { Control-cy3 labeling } \\
\text { efficiency }(\mathrm{pmol} / \mu \mathrm{g})\end{array}$ \\
\hline Fresh 1 & 3.89 & 1.77 & 1.43 & 70.8 & 111 \\
Fresh 2 & 2.94 & 1.86 & 1.79 & 42.8 & 89.6 \\
Fresh 3 & 2.93 & 1.9 & 2 & 52.8 & 97 \\
Mean \pm SD & $3.25 \pm 0.55$ & $1.84 \pm 0.07$ & $1.74 \pm 0.29$ & $55.47 \pm 14.19$ & $99.20 \pm 10.87$ \\
UMFIX 1 & 6.416 & 1.85 & 2 & 78.6 & 110 \\
UMFIX 2 & 2.7138 & 1.86 & 1.84 & 49.6 & 88 \\
UMFIX 3 & 4.012 & 1.86 & 1.8 & 48.2 & 81.8 \\
Mean \pm SD & $4.38 \pm 1.88$ & $1.86 \pm 0.01$ & $1.88 \pm 0.11$ & $58.80 \pm 17.16$ & $93.27 \pm 14.82$ \\
Formalin 1 & 5.366 & 1.58 & 0.84 & 59.8 & 142.4 \\
Formalin 2 & 4.0424 & 1.67 & 1.11 & 41.2 & 73.4 \\
Formalin 3 & 4.427 & 1.93 & 2.08 & 36.2 & 77.4 \\
Mean \pm SD & $4.61 \pm 0.68$ & $1.73 \pm 0.18$ & $1.34 \pm 0.65$ & $45.73 \pm 12.44$ & $97.73 \pm 38.73$ \\
\hline
\end{tabular}


The amplification efficiency of DNA from formalinfixed samples was less than fresh and UMFIX samples, as evidenced by the size and fluorescent intensity of the bands (Fig. 1b).

We also used PCR for GAPDH primers to gauge the quality of extracted DNA. PCR was performed using glyceraldehyde-3-phosphate dehydrogenase primers (GAPDH, Clonetech, Palo Alto, CA) using $0.5 \mu \mathrm{g}$ of RNase-treated isolated DNA and Qiagen TaqPCR Mastermix (Qiagen, Valencia, CA). The conditions for DNA PCR were as follows: $95^{\circ} \mathrm{C}, 15 \mathrm{~min} ; 35$ cycles at $94^{\circ} \mathrm{C}, 45 \mathrm{~s} ; 60^{\circ} \mathrm{C}, 45 \mathrm{~s}$; $72^{\circ} \mathrm{C}, 2 \mathrm{~min}$. As seen in Fig. 1c, a $450 \mathrm{bp}$ band was detected in all samples, although the intensity was considerably lower in formalin-fixed material.

Amplified Genomic DNA was then labeled using BioPrime Array CGH Genomic Labeling kit (Invitrogen, Carlsbad, CA). Quality analysis and quantitation was performed with ND-1000 (NanoDrop Technologies, Wilmington, DE) spectrophotometer to calculate the labeling efficiency. Labeled and purified samples were combined with hybridization master mix and applied to Agilent Human Genome CGH Microarray 44B slides for $40 \mathrm{~h}$ at $65^{\circ} \mathrm{C}$. To minimize the impact of environmental oxidants on signal intensities, slides were scanned immediately using Agilent microarray scanner 4800B. Array images were analyzed using Agilent feature extraction software (v8.1) and CGH explorer (v2.51) [9]. Microarray analytical variables, including the feature signal intensity, data distribution dynamic range, signal to noise ratio and background intensity levels, and the number of saturated and undetected features was used to assess microarray quality.

One of the steps in microarray quality control is labeling efficacy or dye incorporation. Dye incorporation was not drastically different between fresh and UMFIX-exposed samples but formalin-fixed samples showed less dye incorporation (Table 1). There were no differences between fresh and UMFIX-exposed material with regard to scan parameters (Table 2). Formalin-fixed samples, showed various errors such as over-saturation, non-uniformity in replicates and increased signal to noise ratio. The numbers of nonuniform features were at least ten fold higher in formalinfixed samples. The signal to noise ratio of replicated probes can be used to evaluate reproducibility of signals. Formalin-fixed samples showed a higher median \%CV value, indicating lower reproducibility of signal across the microarray and lower signal to noise ratio. The number of features that were saturated in the scanned image was also significantly higher in formalin-fixed samples.

These findings may be attributed to erratic and random fragmentation of DNA in formalin-fixed samples. The fragmentation itself results in increase background noise and aberrant signal intensity due to random hybridization.

We further analyzed array scan data by a-CGH explorer software using stem-plot analysis for moving averages. Fresh and UMFIX-exposed sample showed a readable plot with the expected difference in XY chromosome regions; as expected from the female control and male samples. Also, they showed reproducibly amplified and deleted region in our test sample DNA. A-CGH result from formalin-fixed samples was not interpretable (Fig. 2).

Array $\mathrm{CGH}$ studies have great potential for clinical application. Since the technique does not utilize live cells, it is considerably more advantageous when compared to conventional karyotyping techniques. Array CGH has its own limitations, such as failure to detect translocations; nevertheless it offers unprecedented spatial resolution $[6,11]$. Recently, it has been shown that low-level gene copy number change is associated with changes in expression level of its transcripts [12]. Therefore, results of transcriptomics
Table 2 Representative data of microarray scan quality measurement

Red cy5 labeled samples, green cy3 labeled controls, $18 \mathrm{~h}, 2$ $12 \mathrm{~h}, 324 \mathrm{~h}$

\begin{tabular}{|c|c|c|c|c|c|c|}
\hline \multirow[t]{2}{*}{ Sample } & \multicolumn{2}{|c|}{ Non-uniform feature } & \multicolumn{2}{|c|}{$\begin{array}{l}\text { Reproducibility: non-control } \\
\text { replicated probes median } \% \mathrm{CV}\end{array}$} & \multicolumn{2}{|c|}{ Saturated feature } \\
\hline & Red & Green & Red & Green & Red & Green \\
\hline Fresh 1 & 113 & 379 & 7 & 8 & 0 & 1 \\
\hline Fresh 2 & 51 & 139 & 4 & 4 & 0 & 0 \\
\hline Fresh 3 & 38 & 135 & 5 & 6 & 0 & 0 \\
\hline Mean \pm SD & $67 \pm 40$ & $218 \pm 140$ & $5 \pm 1$ & $6 \pm 2$ & 0 & 0 \\
\hline UMFIX 1 & 352 & 560 & 13 & 13 & 0 & 0 \\
\hline UMFIX 2 & 85 & 179 & 7 & 8 & 0 & 0 \\
\hline UMFIX 3 & 36 & 136 & 6 & 7 & 0 & 0 \\
\hline Mean \pm SD & $158 \pm 170$ & $292 \pm 233$ & $8 \pm 4$ & $9 \pm 3$ & 0 & 0 \\
\hline Formalin 1 & 1,402 & 302 & 77 & 6 & 285 & 1 \\
\hline Formalin 2 & 1,987 & 157 & 37 & 12 & 361 & 0 \\
\hline Formalin 3 & 3,135 & 170 & 94 & 8 & 326 & 0 \\
\hline Mean \pm SD & $2,175 \pm 882$ & $210 \pm 80$ & $69 \pm 30$ & $9 \pm 3$ & $324 \pm 38$ & 0 \\
\hline
\end{tabular}




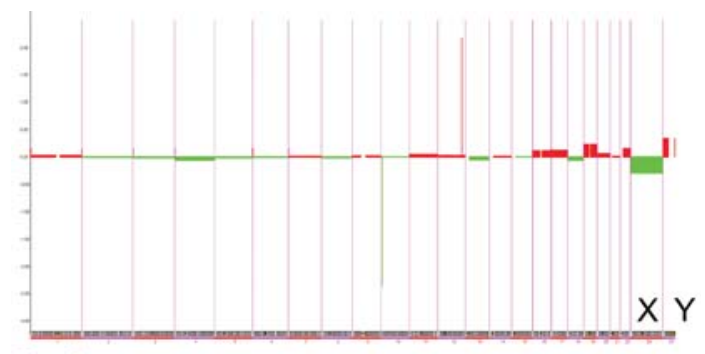

Fresh

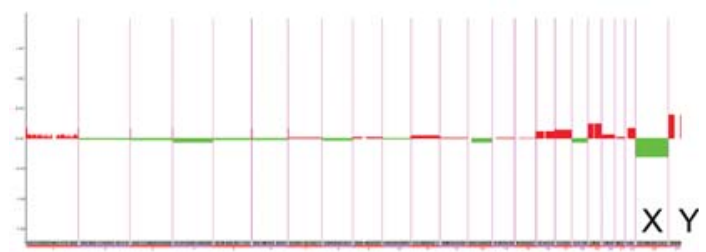

UMFIX

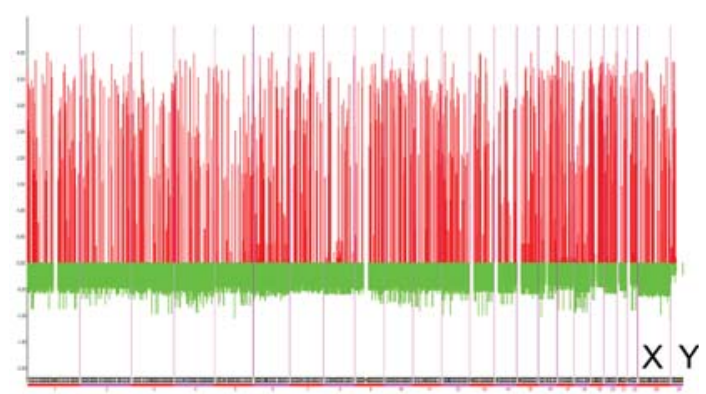

Formalin

Fig. 2 Array CGH stem plot of 8-h preserved skin samples-chromosomes are displayed on $x$-axis and relative ratios on $y$-axis. Green (control XX female, Cy3 labeled) Red (test XY male, Cy5 labeled). $X$ and $Y$ chromosomes are marked

studies can be used to study DNA markers, which are more stable and easier to study than RNA.

Array GGH was originally based on BAC (Bacterial Artificial Chromosomes), but more recently it utilizes oligonucleotides, with its ability to provide whole genomecovering resolution. Synthetic oligonucleotides obviously have the advantage because their exact sequence and length are known for each element on the array. Oligonucleotide array CGH (oa-CGH) has the benefit of overcoming the difficulties inherent to BAC arrays, such as the amount of available DNA, clone management, probe identity due to PCR contaminations, and mapping inaccuracies. Using oa$\mathrm{CGH}$, it is also possible to eliminate another source of error, which is the batch-to-batch variability of Cot-1 DNA, used to block repetitive DNA sequences, since the oligonucleotide probes are designed to be repeat-free [20].

Samples used in nearly all a-CGH studies have been fresh or fresh-frozen tissue. Such material, although useful in research settings, is impractical and cumbersome to use in clinical practice. Furthermore, because diagnostic biopsy samples are relatively small, the amount of residual tissue for additional ancillary testing may be inadequate. Hence there is a great need to develop test strategies that require minimal amounts of tissue and are robust enough to withstand pre-analytical sample preparation. Simplified schemes for sample preservation that allow reliable histomorphology along with preservation of high quality macromolecules are desirable.

We have previously described a novel tissue fixation and processing technique that beside providing adequate histomorphology also preserves high quality HMW RNA, akin to fresh samples [19]. This fixative also protects HMW tissue DNA for use in PCR studies [18]. Here, we further evaluated the suitability of skin tissue DNA for array CGH studies using same methods applied routinely for fresh samples. This study demonstrates that using the novel fixative it is possible to preserve and extract high molecular weight genomic DNA, supported by high labeling efficiency, comparable to that from fresh tissue. Furthermore, no artifacts were seen using this DNA in microarray scanning or analysis for array CGH.

Array CGH studies have been performed on formalinfixed tissues with variable and irreproducible results. Most of the prior studies were based on low-resolution BAC arrays without detailed description of DNA quality or array metrics [10, 21]. Besides, there was often inadequate documentation of fixation time, the volumetric ratio of fixative to tissue, or buffering status. Only few studies have adequately addressed analytical aspects of fixation and processing on array CGH. Ghazani et al. [4] studied the effect of formalin fixation on MCF-7 cell line and on one breast cancer tissue sample. They used two different BAC clone arrays; $1.7 \mathrm{k}$ for the cell line and $19 \mathrm{k}$ for the breast cancer sample [4]. There was no mention of fixative volume or quality of genomic DNA. They showed that long term ( 1 week) fixation results in extensive loss of HMW DNA. In cell lines, the concordance between fresh and formalinfixed samples was around $85 \%$. When genomic DNA was amplified, the concordance decreased to $75 \%$.

Johnson et al. [5] also studied the effect of formalin fixation on a-CGH using BAC arrays. Our results support their conclusion that analysis of DNA samples on agarose gels does not offer any advantage in prediction of suitability of formalin-fixed tissue samples for a-CGH. They also show that the quality of a-CGH depends on the integrity of DNA samples with the requirement that extracted DNA supports the PCR amplification of an amplicon of $300 \mathrm{bp}$ or longer. We showed that it is possible to detect amplicons of up to $450 \mathrm{bp}$ in formalin-fixed sample; (albeit with lower intensity when compared to fresh or UMFIX-exposed samples). The studies by Johnson and others show the possibility of having acceptable results with DNA from formalin-fixed samples using BAC arrays but there are no data that supports its suitability for oa-CGH arrays. This may be due to 
larger sequence of probes presented in BAC array. Other investigators have shown that using alternative approaches to DNA extraction, quantification, amplification or labeling may produce improved results. However, all other authors do agree that the procedures for improving DNA quality are neither substitute for high quality DNA nor they could obtain consistent results from formalin-fixed samples [7, 10]. This is mostly due to complex chemical effect of formalin on tissue, which is still poorly understood. Formalin has a tissue penetration rate of $2.4 \mathrm{~mm}$ in $24 \mathrm{~h}$ and adequate fixation requires at least a ten to one volumetric ratio of fixative to tissue [3]. Therefore, many clinical specimens are only partially fixed by formalin before processing. Furthermore during processing, they are exposed to formalin and alcohol, introducing formalin and alcohol-related tissue artifacts [13]. Formaldehyde is a dipolar molecule and can react with amino, or imino group of the anionic forms of the amino acids. This reaction is time and temperature dependent $[2,9,16,17,18]$. More recent findings show that the deleterious effect of formalin fixation might also result from cumulative action of other reagents and processing conditions. Conversely, no DNA or nucleoside reactions have been reported with ethanol and none would be expected under physiological conditions [8]. By changing the three dimensional structure of the proteins, alcohols prevent protein functions. Therefore, rapid fixation of samples prevents alteration and degradation of biomolecules and preserves them in their native form [11].

In summary, we show that by using a new molecular fixative it is possible to preserve skin tissue DNA that is suitable for array CGH studies; identical to fresh tissue. This can be achieved using same methods and protocols used for fresh samples.

\section{References}

1. Best CJ, Emmert-Buck MR (2001) Molecular profiling of tissue samples using laser capture microdissection. Expert Rev Mol Diagn 1:53-60

2. Feldman NY (1973) Reaction of nucleic acids and nucleoproteins with formaldehyde. Prog Nucleic Acid Res Mol Biol 13:1-49

3. Fox CH, Johnson FB, Whiting J, Roller PP (1985) Formaldehyde fixation. J Histochem Cytochem 33:845-853

4. Ghazani AA, Arneson NC, Warren K, Done SJ (2006) Limited tissue fixation times and whole genomic amplification do not impact array CGH profiles. J Clin Pathol 59:311-315
5. Johnson NA, Hamoudi RA, Ichimura K, Liu L, Pearson DM, Collins VP, Du MQ (2006) Application of array CGH on archival formalin-fixed paraffin-embedded tissues including small numbers of microdissected cells. Lab Invest 86:968-978

6. Kearney L, Horsley SW (2005) Molecular cytogenetics in haematological malignancy: current technology and future prospects. Chromosoma 114:286-294

7. Lee CI, Leong SH, Png AE, Choo KW, Syn C, Lim DT, Law HY, Kon OL (2006) An isothermal method for whole genome amplification of fresh and degraded DNA for comparative genomic hybridization, genotyping and mutation detection. DNA Res 13:77-88

8. Leonov D, Elad D (1977) Light-induced free radical alkylation of polynucleotides and their enzymatic digestion. Nucleic Acids Res 4:319-326

9. Lingjaerde OC, Baumbusch LO, Liestol K, Glad IK, BorresenDale AL (2005) CGH-explorer: a program for analysis of arrayCGH data. Bioinformatics 21:817-820

10. Little SE, Vuononvirta R, Reis-Filho JS, Natrajan R, Iravani M, Fenwick K, Mackay A, Ashworth A, Pritchard-Jones K, Jones C (2006) Array CGH using whole genome amplification of freshfrozen and formalin-fixed, paraffin-embedded tumor DNA. Genomics 87:298-306

11. Pinkel D, Albertson DG (2005) Comparative genomic hybridization. Annu Rev Genomics Hum Genet 6:331-354

12. Pollack JR, Sorlie T, Perou CM, Rees CA, Jeffrey SS, Lonning PE, Tibshirani R, Botstein D, Borresen-Dale AL, Brown PO (2002) Microarray analysis reveals a major direct role of DNA copy number alteration in the transcriptional program of human breast tumors. Proc Natl Acad Sci USA 99:12963-12968

13. Rait VK, O'Leary TJ, Mason JT (2004) Modeling formalin fixation and antigen retrieval with bovine pancreatic ribonuclease A: I-structural and functional alterations. Lab Invest 84:292-299

14. Shaffer LG, Bejjani BA (2004) A cytogeneticist's perspective on genomic microarrays. Hum Reprod Update 10:221-226

15. Smeets DF (2004) Historical prospective of human cytogenetics: from microscope to microarray. Clin Biochem 37:439-446

16. Theis ER (1944) The protein-formaldehyde reaction. I Collagen. J Biol Chem 154:87-97

17. Theis ER (1945) The protein-formaldehyde reaction. III. The effect of temperature and denaturation. J Biol Chem 157:7-14

18. Vincek V, Nassiri M, Nadji M, Morales AR (2003) A tissue fixative that protects macromolecules (DNA, RNA, and protein) and histomorphology in clinical samples. Lab Invest 83:1427-1453

19. Vincek V, Nassiri M, Block N, Welsh CF, Nadji M, Morales AR (2005) Method for extraction of high quality RNA from laser capture microdissected H\&E stained paraffin sections. Diagn Mol Pathol 14:127-133

20. Ylstra B, van den Ijssel P, Carvalho B, Brakenhoff RH, Meijer GA (2006) BAC to the future! or oligonucleotides: a perspective for micro array comparative genomic hybridization (array $\mathrm{CGH}$ ). Nucleic Acids Res 34:445-450

21. Zielenska M, Marrano P, Thorner P, Pei J, Beheshti B, Ho M, Bayani J, Liu Y, Sun BC, Squire JA, Hao XS (2004) High-resolution cDNA microarray CGH mapping of genomic imbalances in osteosarcoma using formalin-fixed paraffin-embedded tissue. Cytogenet Genome Res 107:77-82 